\title{
Familial inflammatory inclusion body myositis
}

\author{
B Ranque-Francois, T Maisonobe, E Dion, J-C Piefte, M-P Chauveheid, Z Amoura, T Papo
}

Ann Rheum Dis 2005;64:634-637. doi: 10.1136/ard.2004.025494

\begin{abstract}
Objective: To compare familial inflammatory inclusion body myositis (IBM) with hereditary inclusion body myopathies and sporadic IBM.

Patients and methods: Clinical, biological, MRI, and histological data were analysed in two siblings with inflammatory IBM and compared with those of patients with sporadic and hereditary IBM.

Results: Both patients had a clinical phenotype of sporadic IBM, which differs from hereditary myopathies because of late age of onset-respectively 65 and 66 years, and different pattern of muscular involvement-asymmetric, mainly distal but also involving quadriceps. MRI showed selective fatty infiltration and oedema in the extensor compartment of thigh muscles. The diagnosis of IBM was confirmed by muscle biopsy, showing muscle fibres containing numerous rimmed vacuoles, a characteristic shared by all types of IBM. In contrast with hereditary IBM, histological analysis also showed inflammatory mononuclear infiltrate invading non-necrotic fibres, ragged red and oxidase c negative fibres, and positive Congo red staining. Moreover, HLA class II typing disclosed DR $\beta 10301$ haplotype, which is significantly related to sporadic but not to hereditary IBM. With steroid treatment and monthly intravenous immunoglobulins, the disease was stabilised in both patients at protracted follow up.

Conclusion: Sporadic and familial inflammatory IBM share the same clinical, biological, MRI, and histological features.
\end{abstract}

$\mathrm{S}$ poradic inclusion body myositis (IBM) is the most common muscle disease in the elderly. It is characterised by a distal and proximal myopathy, progressively leading to severe disability. Muscle biopsy shows abnormal muscle fibres containing vacuoles and typical filamentous inclusions, with lymphocytic inflammation. In 1993, the term hereditary IBM was introduced to designate hereditary muscle diseases with histology resembling sporadic IBM, but without inflammation. That term encompasses several syndromes with an early age of onset and various clinical presentations. Unlike hereditary IBM, inflammatory IBM typically occurs sporadically. We report here two cases of familial inflammatory IBM.

\section{CASE REPORTS}

\section{Case 1}

A white woman aged 67, native of Guadeloupe, French Antillas, was referred to our department in January 1998. She was born in a non-consanguineous family of seven siblings (fig 1). Her history included a breast carcinoma in 1995, which required mastectomy and chemotherapy.

Eight months after surgery, a weakness in the lower limbs occurred: she could hardly rise from chairs, climb stairs, and walk for more than 10 minutes. Electrophysiological studies showed a myopathic pattern. The symptoms improved within a few months without specific treatment.

In September 1997 the weakness worsened, leading to frequent falls, and extended to the upper extremities. Physical examination showed a major atrophy of quadriceps, calves, and forearms. The following muscle grades were noted: neck flexors 4 , neck extensors 8 , quadriceps 4 on the left and 5 on the right, hip flexors 8 , hip abductors 8 , hip extensors 8, ankle dorsiflexors 3 on the left and 6 on the right, ankle plantar flexors 5 on the left and 4 on the right, shoulder abductors 10, elbow flexors 9, elbow extensors 6 on the left and 8 on the right, wrist flexors 5 , wrist extensors 6 on the left and 8 on the right, grip 4 on the left and 8 on the right. To sum up, motor impairment was bilateral but asymmetric, mainly distal, with a striking waste of quadriceps. There were neither dysphagia nor respiratory symptoms. Sensation and tendon reflexes were normal.

Blood creatine kinase level was $820 \mathrm{IU} / \mathrm{l}$ (normal <210). Viral serologies, including HIV and HTLV1, and serum autoantibodies were negative. HLA class II typing was DRß1 0301, DRß1 01. Magnetic resonance imaging (MRI) showed a marked muscle wasting and a fatty infiltration. The quadricipital group of thighs was predominantly affected as compared with the flexor compartment (fig 2). Electromyography confirmed a pure myopathic pattern. Muscle biopsy showed red-rimmed vacuoles in some atrophic fibres and inflammatory mononuclear infiltrate in or around muscle fibres, with few intracellular deposits on Congo red staining, therefore highly suggestive of sporadic IBM. Widespread expression of HLA class I was seen on muscle fibres.

Steroid treatment was started: muscle weakness remained but creatine kinase normalised in January 2000. In November 2000, high dose intravenous immunoglobulin (IVIg) treatment was added because some episodes of choking had occurred. Thirty nine months later, muscle strength remains unchanged.

\section{Case 2}

In April 2000, one of the brothers of patient 1, aged 66, a farmer in Guadeloupe, was referred to our centre because lower limb weakness had insidiously developed, leading to difficulty in climbing steps, and falls.

Physical examination showed atrophy of quadriceps and forearms. Bilateral motor impairment affected both proximal and distal muscles in the lower limbs, but only wrist flexors in the upper extremities. Muscle grades were as follow: neck flexors 8 , neck extensors 10, quadriceps 8 on the left and 6 on the right, hip flexors 7, hip abductors 10, hip extensors 10 , ankle dorsiflexors 8 on both sides, ankle plantar flexors 6 on the left and 8 on the right, shoulder abductors 10, elbow flexors 6 on the left and 8 on the right, elbow extensors 10 , wrist flexors 8 on the left and on the right, wrist extensors 10 on the left and 8 on the right, grip 6 on the left and 7 on the

Abbreviations: IBM, inclusion body myositis; IVlg, intravenous immunoglobulin; MRI, magnetic resonance imaging 


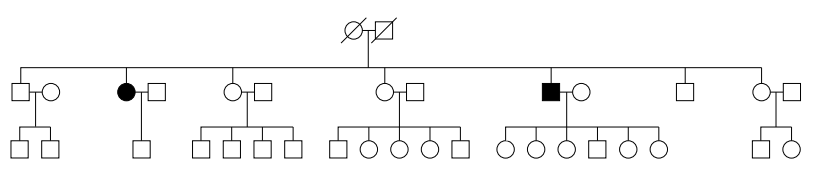

Figure 1 Pedigree of the family.

right. Sensation and tendon reflexes were normal. There were no respiratory symptoms, but some swallowing difficulties had occurred.

The blood creatine kinase level was normal, viral serologies and serum autoantibodies were negative. HLA class II typing was DR $\beta 1$ 0301, DR $\beta 101$ and DQ $\beta 1$ 0201, DQ $\beta 105011$. Electromyography showed a pure myopathic pattern in the distal and proximal muscles of the lower limbs and in distal muscles of the upper limbs.

Muscle biopsy disclosed many necrotic or non-necrotic fibres surrounded and/or invaded by numerous lymphocytes and monocytes-macrophages, without any red-ragged fibres (fig 3). Numerous rimmed vacuoles were visible in patches of atrophic fibres. Congo red staining was positive in a few fibres. HLA class I molecules were overexpressed on muscle fibres. High dose IVIg infusions and steroid treatment were started in May 2000. The patient felt a clear subjective improvement from the third infusion, but muscle testing has remained unchanged after 45 infusions.

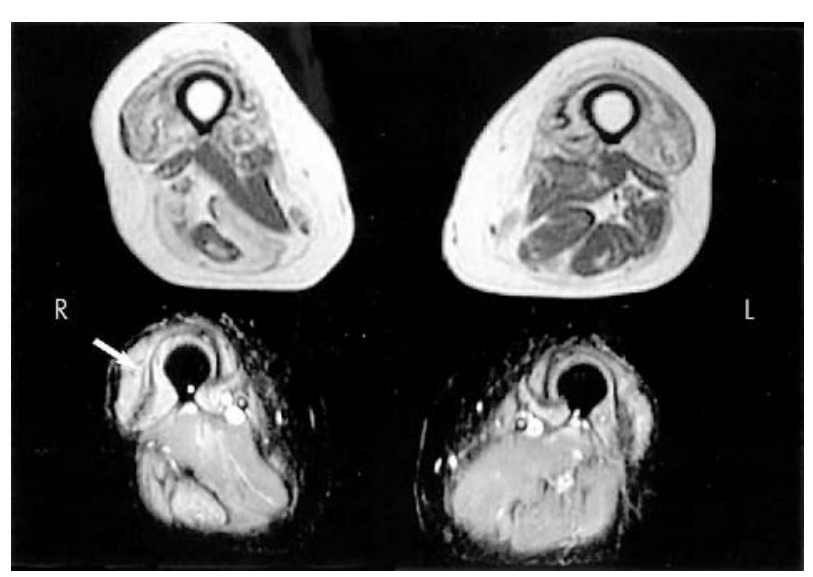

Figure 2 Axial spin echo $T_{1}$ and STIR images through the mid-portion of the two thighs (patient 1). Spin echo $T_{1}$ image (upper) shows fatty infiltration (high signal intensity areas) predominant in both quadricipital groups and in the posterior group of the right thigh. STIR image (lower) demonstrates oedema mostly in the quadricipital group (arrow).

\section{DISCUSSION}

Sporadic IBM is a chronic, acquired inflammatory myopathy, which clearly differs from dermatomyositis and polymyositis. It usually develops after the age of 50 years, more commonly affecting men, and is characterised by progressive asymmetric, proximal and distal weakness that early on involves the quadriceps and volar forearm muscles (wrist and finger flexors). Dysphagia occurs in two thirds of the cases. Spontaneous evolution of muscle weakness is slow but patients become severely disabled, usually confined to a wheelchair within 10 years. ${ }^{12}$ Death may occur, mainly from choking. Electromyography generally shows myopathic motor unit potentials and may also reveal a mixed myopathic-neuropathic pattern. Serum creatine kinase level is normal or mildly increased. Muscle MRI typically shows atrophy and fatty degeneration in $\mathrm{T}_{1}$ weighted scans and increased signal intensity in $\mathrm{T}_{2}$ weighted scans. Muscle biopsy shows scattered muscle fibres containing red-rimmed vacuoles (basophilic granular inclusions distributed around the edge of slit-like vacuole) within cytoplasm and nuclei, and eosinophilic cytoplasmic inclusions. Inflammation is associated with partial invasion of non-necrotic fibres by activated CD8 T lymphocytes and macrophages. Additionally, muscle fibres are pathologically expressing HLA class I molecules. Ragged red fibres and cytochrome $c$ oxidase negative fibres are often seen. Small intracellular amyloid deposits are present on Congo red stain in $80 \%$ of cases. Amyloid deposits are composed of the same proteins seen in Alzheimer brain deposits. Electron microscopy shows the accumulation of 15-21 nm tubulofilaments in cytoplasm or myonuclei. ${ }^{1}$

In contrast, hereditary IBM is a heterogeneous group of non-inflammatory, red-rimmed vacuolar myopathies, composed of different families with various clinical phenotypes. The onset occurs earlier in life (20-30 years), and the pattern of weakness is quite different from sporadic IBM. ${ }^{3}$ Two modes of inheritance are observed. Autosomal recessive hereditary IBM usually results in distal weakness of the lower limbs, sparing the quadriceps even when proximal muscles are affected, and progresses very slowly. Recently, the disease was related to several mutations in the GNE gene on chromosome 9. ${ }^{4}$ Autosomal dominant hereditary IBM is characterised by a limb-girdle distribution of muscle weakness with a quicker deterioration, but probably reflects heterogeneous genetic defects. ${ }^{5}$ Compared with sporadic IBM, muscle histology also shows red-rimmed vacuoles and accumulation of amyloid proteins, but with much lower Congo red staining. Additionally, there are neither ragged red fibres nor cytochrome $c$ oxidase negative fibres. The principal distinctive feature is the absence of significant inflammatory infiltrate in the endomysium, around non-necrotic muscle fibres. ${ }^{1}$
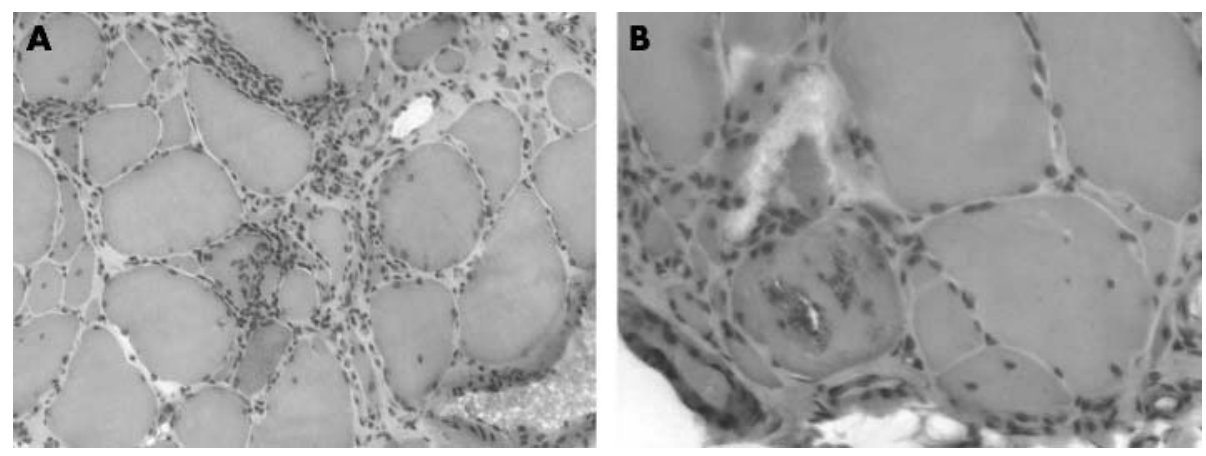

Figure 3 Cryostat anterior tibial muscle biopsy specimen showing (A) non-necrotic fibres surrounded by mononuclear inflammatory cells at the endomysium and perimysium; (B) typical red-rimmed vacuoles. Light microscopy. Haematoxylin and eosin $\times 250$ and $\times 400$. 
Table 1 Characteristics of published familial cases of IBM

\begin{tabular}{|c|c|c|c|c|c|c|c|c|c|c|c|c|}
\hline \multirow[b]{2}{*}{ Reference } & \multicolumn{4}{|c|}{ Clinical features } & \multirow[b]{2}{*}{ CK } & \multirow[b]{2}{*}{ EMG } & \multicolumn{4}{|l|}{ Histology } & \multirow{2}{*}{$\begin{array}{l}\text { MRI: atrophy } \\
\text { +fatty } \\
\text { degeneration }\end{array}$} & \multirow[b]{2}{*}{ Treatmen } \\
\hline & $\begin{array}{l}\text { Age at } \\
\text { onset }\end{array}$ & Sex & Atrophy & Weakness & & & $\begin{array}{l}\text { Rimmed } \\
\text { vacuoles }\end{array}$ & $\begin{array}{l}\text { Mononuclear } \\
\text { inflammation }\end{array}$ & $\begin{array}{l}\text { Congo red } \\
\text { staining }\end{array}$ & $\begin{array}{l}\text { Tubulofilament } \\
18-21 \mathrm{~nm}\end{array}$ & & \\
\hline \multirow[t]{2}{*}{ Nauman $^{8}$} & 55 & $\mathrm{~F}$ & $Q, T A$ & $P D^{*} d$ & $6 \mathrm{~N}$ & My & + & + & NA & + & $Q$, left TA & $\mathrm{C}$ \\
\hline & 48 & $\mathrm{~F}$ & $Q, T A$ & $P D^{*} d$ & $4 \mathrm{~N}$ & My & + & + & NA & + & NA & C \\
\hline Sivakumar $^{7}$ & 73 & $M$ & $Q, V F$ & PDS & $1 \mathrm{~N}$ & NA & + & + & + & + & NA & NA \\
\hline Family A & 69 & $M$ & $Q>V F$ & PD & $1.5 \mathrm{~N}$ & NA & + & + & + & + & NA & NA \\
\hline \multirow[t]{2}{*}{ Family B } & 55 & $M$ & $\mathrm{Q}<\mathrm{VF}$ & PD & $2 \mathrm{~N}$ & NA & + & + & + & + & NA & NA \\
\hline & 55 & $M$ & $\hat{Q}>V F$ & PD & $3 \mathrm{~N}$ & NA & + & + & + & + & NA & NA \\
\hline \multirow{3}{*}{ Family C } & 60 & $M$ & $Q>V F$ & PD d & $1 \mathrm{~N}$ & NA & + & + & + & + & $Q>T F$ & NA \\
\hline & 60 & $M$ & $Q>V F$ & PD & $1.5 \mathrm{~N}$ & NA & + & + & + & + & $Q+T F$ & NA \\
\hline & 48 & $\mathrm{~F}$ & $Q, V F$ & PD & $1 \mathrm{~N}$ & NA & NA & NA & NA & NA & $Q>T F$ & NA \\
\hline \multirow[t]{2}{*}{ Amato $^{6}$} & 62 & $M$ & $Q, V F$ & $P D d$ & $2 \mathrm{~N}$ & My & + & + & + & + & NA & NA \\
\hline & 60 & $M$ & $Q, V F$ & PDS & NA & My & + & + & + & NA & NA & NA \\
\hline \multirow{2}{*}{ Personal report } & 64 & $\mathrm{~F}$ & $Q, V F$ & PDd & $2 \mathrm{~N}$ & My & + & + & + & NA & $Q>T F$ & $C+\lg I V$ \\
\hline & 65 & $M$ & $Q>V F$ & $P D d$ & $1 \mathrm{~N}$ & My & + & + & + & NA & NA & $C+$ IgIV \\
\hline
\end{tabular}

*Hand and finger extensors more severely affected than flexors.

CK, creatine kinase; EMG, electromyography; MRI, magnetic resonance imaging; $Q$, quadriceps; TA, tibialis anterior; $P$, proximal; $D$, distal; $d$, dysphagia; My, myopathic; NA, not available; C, corticosteroids; VF, volar forearm; S, sensitive anomalies; TF, thigh flexors.

Our patients had a clearcut phenotype of sporadic IBM because of $(a)$ the late age of onset; $(b)$ the selective pattern of clinical involvement, affecting the quadriceps and the long finger flexor muscles; and (c) typical histological features. Because the prevalence of sporadic IBM is about $1 / 100000$, the probability of observing two cases in the same family is extremely low. Still, five families with at least two affected siblings have been reported in English publications so far. ${ }^{6-9}$ Table 1 reports the clinical, biological, histological, and MRI characteristics of these patients; they do not differ from the usual characteristics of sporadic IBM. The only exception is the family reported by Naumann, where onset was earlier, with moderate atrophy of the quadriceps and volar forearms, and prominent weakness in finger and arm extensors. ${ }^{8}$

No significant relation with HLA phenotype has been found in patients with hereditary IBM. On the contrary, Garlepp et al found DR52 and DR3 genotype in respectively $100 \%$ and $92 \%$ of patients with sporadic IBM. ${ }^{10}$ Another study found $77 \%$ of DR $\beta 1$ 0301, DR $\beta 3$ 0101, or DR $\beta 30202$ alleles (DR3 haplotype) and 70\% of DQ $\beta 10201$ in sporadic IBM, which confirms the association with HLA class II. ${ }^{11}$ In familial inflammatory IBM, the DR $\beta 10301$ allele was found in our two patients and in every affected member of the families described by Sivakumar. ${ }^{7}$ Patient 2 was also DQ $\beta 1$ 0201 (DQ typing is not available for the other patient). HLA phenotype was not specified in the other reports of familial inflammatory IBM. These HLA class II alleles, which are present in only $15-20 \%$ of a white control population, are also highly prevalent in polymyositis, dermatomyositis, and other inflammatory diseases.

Despite recent progress in understanding the pathogenesis of sporadic IBM, the early mechanisms remain unclear. Several studies provide evidence for an overexpression of $\beta$ amyloid protein precursor, as well as other proteins seen in the $\beta$-amyloid of Alzheimer's disease (chymotrypsin, apoE, phosphorylated $\tau \ldots$ ), possibly leading to oxidative stress. ${ }^{1}$ The other striking feature is the presence of activated cytotoxic $\mathrm{T}$ cells surrounding healthy, but HLA-I class expressing, muscle fibres. Interestingly, their T cell receptor repertoire seems to be oligoclonal, which suggests a clonal expansion of the CD8+ cells by a still unknown superantigen. ${ }^{12}$

In contrast with dermatopolymyositis or polymyositis, no treatment seems to be effective in sporadic IBM. Steroids do not provide measurable benefit. IVIg showed non-significant improvement in global muscle strength in four controlled clinical trials, ${ }^{13-15}$ except for swallowing muscles in one series. ${ }^{14}$ This treatment might therefore be considered when oesophageal impairment occurs. In familial inflammatory IBM, reported data about treatment are not available, except for one atypical patient who responded to glucocorticoids. ${ }^{8}$ At protracted follow up, muscle disease stabilised in both our patients with prolonged low dose steroids and IVIg treatment.

In conclusion, sporadic and familial inflammatory IBM share the same clinical, biological (including HLA class II genotype), MRI, and histological features.

\section{Authors' affiliations}

B Ranque-Francois, M-P Chauveheid, T Papo, Internal Medicine Department, La Pitié-Salpétrière Hospital, 83 Boulevard de l'Hôpital, 75651 Paris Cedex 13, France

T Maisonobe, Neuropathology Department, La Pitié-Salpétrière Hospital, 83 Boulevard de l'Hôpital, 75651 Paris Cedex 13, France E Dion, Radiology Department, La Pitié-Salpétrière Hospital, 83 Boulevard de l'Hôpital, 75651 Paris Cedex 13, France

J-C Piette, Z Amoura, Internal Medicine Department, La Pitié-Salpétrière Hospital, 83 Boulevard de l'Hôpital, 75651 Paris Cedex 13, France

Correspondence to: Professor T Papo, thomas.papo@bch.ap-hop-paris. $\mathrm{fr}$

Accepted 22 August 2004

\section{REFERENCES}

1 Askanas V, Engel WK. Sporadic inclusion-body myositis and hereditary inclusion body-myopathies: diagnosis and pathogenesis. Curr Opin Rheumatol 1998; 10:530-42.

2 Dalakas MC. Understanding the immunopathogenesis of inclusion body myositis: present and future prospects. Rev Neurol 2003; 158:948-58.

3 Tome FM, Fardeau M. Hereditary inclusion body myopathies. Curr Opin Neurol 1998;11:453-9.

4 Eisenberg I, Avidan N, Potikha T, Hochner H, Chen M, Olender T, et al. The UDP-N-acetylglucosamine 2-epimerase/ $\mathrm{N}$-acetylmannosamine kinase gene is mutated in recessive hereditary inclusion body myopathy. Nat Genet 2001;29:83-7.

5 Argov Z, Eisenberg, Mitrani-Rosenbaum S. Genetics of inclusion body myopathies. Curr Opin Rheumatol 1998;10:543-7.

6 Amato AA, Shebert RT. Inclusion body myositis in twins. Neurology 1998;51:598-600.

7 Sivakumar K, Semino-Mora C, Dalakas MC. An inflammatory, familial, inclusion body myositis with autoimmune features and a phenotype identical to sporadic inclusion body myositis. Study in three families. Brain 1997; 120:653-61.

8 Naumann NM, Reichmann H, Goebel HH, Moll C, Tockya KV. Glucocorticoid-sensitive hereditary inclusion body myositis. J Neurol 1996;243:126-30.

9 Rider LG, Gurley RC, Pandey JP, de la Torre IG, Kalovidouris AE, $\mathrm{O}^{\prime}$ Hanlon TP, et al. Clinical, serologic, and immunogenetic features of 
familial idiopathic, inflammatory myopathy. Arthritis Rheum 1998;41:710-19.

10 Garlepp MJ, Laing B, Zilko J, Ollier W, Mastaglia FL. HLA associations with inclusion body myositis. Clin Exp Immunol 1994:98:40-5.

11 Koffman BM, Sivakumar K, Simonis T, Stroncek D, Dalakas MC. HLA allele distribution distinguishes sporadic inclusion body myositis from inclusion body myopathies. J Neuroimmunol 1998;842:139-42.

12 Fyhr IM, Moslemi AR, Mosavi AA, Lindberg C, Tarkowski A, Oldfors A. Oligoclonal expansion of muscle infiltrating T cells in inclusion body myositis. $J$ Neuroimmunol 1997;9:185-9.
13 Dalakas MC, Koffman B, Fujii M, Spector S, Sivakumar K, Cupler E. A controlled study of intravenous immunoglobulin combined with prednisone in the treatment of inclusion body myositis. Neurology 2001:56:323-7.

14 Dalakas MC. Controlled studies with high-dose intravenous immunoglobulin in the treatment of dermatomyositis, inclusion body-myositis and polymyositis. Neurology 1998;51(suppl 5):S37-45.

15 Walter MC, Lochmuller H, Toepfer M, Schlotter B, Reilich P, Schroder M, et al. High dose immunoglobulin treatment in sporadic inclusion body myositis: a controlled study. J Neurol 2000;247:22-8.
Get published within days of acceptance with ARD

We are delighted to announce that the Annals of the Rheumatic Diseases launched a "publish ahead of print" programme in February 2004. Selected papers are fast tracked and published online months before they appear in the print journal.

Papers of major significance to the international rheumatology community are published within days of acceptance. The first published article is the raw accepted manuscript; edited and typeset versions are also published as soon as they are available.

In addition to being available on ARD Online, the publish ahead of print articles are searchable through PubMed/ Medline-establishing primacy for your work. They are linked from the ARD Online home page.

$A R D$ 's publish ahead of print programme is unique among the major rheumatology journals - to take advantage of this service submit your papers to Annals of the Rheumatic Diseases using our online submission and review system Bench>Press (http://submit-ard. bmijournals.com). For further information contact ARD@bmigroup.com. 